\title{
Factores personales e institucionales relacionados a la calidad de las anotaciones de enfermería en el servicio de cirugía del Hospital Guillermo Almenara Irigoyen, abril-junio, 2011
}

\author{
Carmen Rosa BARTOLO ESTRELLA', Mary Luz SOLÓRZANO APARICIO²
}

\begin{abstract}
RESUMEN
Objetivo: Determinar cuál es la relación entre los factores personales e institucionales con la calidad de las anotaciones de enfermería del servicio de cirugía del Hospital Nacional Guillermo Almenara Irigoyen, abril-junio del 2011. Metodología: El estudio fue de tipo correlacional de corte transversal. La población del presente estudio estuvo conformada por 40 anotaciones de enfermería del servicio de cirugía, que fueron escritos por licenciadas en enfermería y de pacientes que ingresaron la primera semana del mes de junio del 2011, y 20 licenciadas en enfermería del servicio de cirugía. La muestra es no probabilística por conveniencia. Resultados: En este estudio se reportó que el $50 \%$ de factores institucionales desfavorecen en la elaboración de las anotaciones de enfermería, el $45 \%$ de factores personales favorecen en la elaboración de las anotaciones de enfermería y el 57.5\% de anotaciones son de regular calidad. Conclusiones: El estudio concluye que la mayoría de factores institucionales desfavorecen en la elaboración de las anotaciones de enfermería, y esto se evidencia en las anotaciones de enfermería de regular calidad.
\end{abstract}

Palabras clave: Anotaciones de enfermería, factores personales e institucionales, calidad.

\begin{abstract}
Objective: The overall objective of the research was to determine the level of knowledge, attitudes and practices of the bioethical principles of professional nursing in patient care in the surgery department. Methodology: This study is a quantitative approach, non-experimental design and scope exploratory, descriptive. The population consisted of nurses working in the health care area of General Surgery which represent a total of 20 nurses; it is considered $100 \%$ of the population. Data were collected using three instruments: a multiple choice questionnaire to measure the level of knowledge, the scale type Likerth to measure the attitudes and an observation guide for measuring the level of practice. Results: The $60 \%$ of nurses have a good level of knowledge, while only $15 \%$ an outstanding level of knowledge. Also, about the level of attitude, $80 \%$ of nurses has a level of positive attitude. In the level of practice, $60 \%$ of the population put in practice all bioethical principles during patient care, collaborating on improving the quality life of the patient as far as possible giving excellent physical care, mental and spiritual. Conclusion: There is a significant correlation between the level of knowledge and practice $(\mathrm{r}=0.526, \mathrm{p}$ valor 0.017$)$. The interaction between knowledge, attitudes and practices, are expressed in health are among social settings, and contribute to the technical, scientific and humanistic development of the profession.
\end{abstract}

Keywords: Nursing ethics, bioethic, bioethical principles, nursing care, surgery.

\footnotetext{
1 Estudiante del $5^{\circ}$ año de Enfermería, Universidad Peruana Unión.

${ }^{2}$ Magíster en Ciencias, con mención en Enfermera Educadora, Docente de la Facultad de Ciencias de la Salud, Universidad Peruana Unión
} 


\section{INTRODUCCIÓN}

Las actividades y observaciones de Enfermería son una preocupación constante en nuestra sociedad. Desde el punto de vista histórico, las enfermeras han tenido una difícil relación con los registros de enfermería. A pesar de que la cantidad de los registros de enfermería ha aumentado ciertamente con los años, no se puede decir lo mismo de la calidad de la información registrada. Las anotaciones de enfermería son variadas, complejas y consumen tiempo. Estudios realizados reflejan que las enfermeras pasan entre 35 y 140 minutos escribiendo sus informes después de cada turno (García, 2003).

Se realizó un estudio en Sao Paulo, Brasil sobre "Evaluación de la calidad de la documentación de enfermería en cuanto a la revisión de registros médicos del paciente": "En el análisis cualitativo de los registros de enfermería fueron $26,7 \%$ considerados pobres, el $64,6 \%$ fueron regulares y el $8,7 \%$ buena. No hubo archivos evaluados como excelentes. En general, los problemas encontrados en los registros de enfermería fueron: el desarrollo de las notas de enfermería y notas indefinidas en cuanto al contenido. También hubo ilegibilidad, errores de ortografía, uso incorrecto de la terminología y siglas no estandarizadas y sin hacer referencia a algunos de los registros locales, así como fallas en la identificación, ya sea por falta de sello o nombres ilegibles". (Grespan \& D'Innocenzo, 2007).

En el Perú este problema no es una excepción ya que varios trabajos de investigación reflejan la presencia de este problema.

Fretel (2002), en su trabajo de investigación sobre Evaluación de las Anotaciones de Enfermería durante los Años 2000-2002 en el Servicio de Neurología del Hospital Nacional Guillermo Almenara Irigoyen, llegó a la conclusión de que "Las Anotaciones de Enfermería durante los años 2000-2002 son en un $50 \%$ medianamente significativas, evidenciando que el cuidado brindado no ha sido integral. En la elaboración de las anotaciones de enfermería encontramos que solo el $21.8 \%$ es calificada como adecuada, lo cual indica que en más de la mitad de la población en estudio no considera entre sus criterios de elaboración datos importantes en base al PAE, lo cual no nos permitiría valorar, en su mayoría, el cuidado brindado al paciente".

Betancourt, Figueredo y Uribe (1998), en su trabajo de investigación sobre Calidad de las anotaciones de enfermería en un Servicio de Medicina del Hospital Nacional Arzobispo Loayza, llegaron a la conclusión que las anotaciones son de regular calidad, $37.3 \%$ en el turno diurno, $40.9 \%$ en el turno nocturno debido a que no consideran en su totalidad los ítems evaluados.

Se observa que la elaboración de las anotaciones de enfermería no son de calidad, lo cual se evidencia en los trabajos de investigación anteriormente citados, demostrándose la problemática real de los registros, considerando que éstos tienen gran importancia en la prestación y continuidad de cuidados, siendo que constituyen un importante documento legal.

La organización del trabajo para la atención continua en los servicios hospitalarios se ha establecido en turnos, de manera que el paciente y familia son atendidos por diferentes profesionales, lo que hace imprescindible el uso de registros sobre los cuidados que brinda el personal de enfermería. Es así como los registros son canales de comunicación entre los diferentes profesionales y facilitan la toma de decisiones en forma oportuna para contribuir a la atención continua.

Un buen registro de enfermería debería ser aquel que recogiese la información suficiente como para permitir que otro profesional, de similar calificación, asumiera sin dificultad la responsabilidad del cuidado del paciente (Amezcua, 1995).

Este marco referencial generó interés durante las prácticas clínicas que se realizaron en la Clínica San Isidro Labrador, al ver que las anotaciones de enfermería eran repetitivas, tenían escasa información y no seguían un modelo. Esto originó la idea de realizar este estudio, con el objetivo principal de determinar la relación entre los factores personales e institucionales con la calidad de las anotaciones de enfermería del servicio de Cirugía del Hospital Nacional Guillermo Almenara Irigoyen.

\section{MATERIAL Y MÉTODOS}

Estudio de diseño no experimental, de tipo correlacional de corte transversal porque se recolectó los datos en un solo momento y en un tiempo único; y de tipo correlacional, porque se relacionaron dos variables: factores personales e institucionales con la calidad de las anotaciones de enfermería.

La población del presente estudio estuvo conformada por 40 anotaciones de enfermería del servicio de cirugía que fueron escritos por licenciadas de enfermería y de pacientes que ingresaron la primera semana del mes de junio del 2011 y por 20 licenciadas del servicio de cirugía. El muestreo fue no probabilístico por conve- 
niencia.

Se utilizó una lista de chequeo para evaluar las anotaciones de enfermería y el cuestionario para evaluar los factores personales e institucionales del personal de enfermería.
Los datos fueron transferidos y procesados en el software estadístico SPSS 15, se realizó el análisis descriptivo de la variable anotaciones de enfermería y se usó la prueba chi cuadrado para relacionar las variables en estudio.

\section{RESULTADOS}

\section{Tabla 1}

Factores institucionales y el nivel de calidad de las anotaciones de enfermería del servicio de cirugía del HNGAI, Abril-Junio 2011

\begin{tabular}{cccccc}
\hline & & & Factores institucionales & Total \\
& & & favorecen & desfavorecen & 5 \\
\hline \multirow{2}{*}{$\begin{array}{c}\text { Nivel de calidad de } \\
\text { las anotaciones de }\end{array}$} & calidad & $\mathrm{N}$ & 3 & 2 & 25 \\
enfermería del servicio & regular calidad & $\mathrm{N}$ & 2 & 10 & 12 \\
de cirugía del HNGAI, & & $\%$ & 10 & 10 & 60 \\
Junio 2011 & mala calidad & $\mathrm{N}$ & 0 & 50 & 3 \\
& & $\%$ & 0 & 3 & 15 \\
Total & & $\mathrm{N}$ & 5 & 15 & 20 \\
& & $\%$ & 25 & 75 & 100 \\
\hline
\end{tabular}

En cuanto a la influencia de los factores institucionales en la elaboración de las anotaciones de enfermería, en la tabla 1, se puede apreciar que el 50\% de factores institucionales desfavorece en la elaboración de las anotaciones de enfermería, esto se evidencia en las anotaciones de enfermería de regular calidad.

\section{Tabla 2}

\section{Factores personales y el nivel de calidad de las anotaciones de enfermería del servicio de cirugía del HNGAI, Abril-Junio 2011}

\begin{tabular}{cccccc}
\hline & & & Factores personales \\
& & & favorecen & Tosfavorecen & Total \\
\hline & calidad & $\mathrm{N}$ & 4 & 1 & 5 \\
Nivel de calidad de & $\%$ & 20 & 5 & 25 \\
$\begin{array}{c}\text { las anotaciones de } \\
\text { enfermería del servicio } \\
\text { de cirugía del HNGAI, }\end{array}$ & regular calidad & $\mathrm{N}$ & 9 & 3 & 12 \\
Junio 2011 & & $\%$ & 45 & 15 & 60 \\
& mala calidad & $\mathrm{N}$ & 3 & 0 & 3 \\
Total & $\%$ & 15 & 0 & 15 \\
& & $\mathrm{~N}$ & 16 & 4 & 20 \\
\hline
\end{tabular}

En cuanto a la influencia de los factores personales en la elaboración de las anotaciones de enfermería, en la tabla 2 , se puede apreciar que el $45 \%$ de factores personales favorece en la elaboración de las anotaciones de enfermería, esto se evidencia en las anotaciones de enfermería de regular calidad. 
Tabla 3

Nivel de calidad de las anotaciones de enfermería del servicio de cirugía del Hospital Nacional Guillermo Almenara Irigoyen, Junio 2011.

\begin{tabular}{ccc}
\hline & $\mathrm{N}$ & $\%$ \\
\hline calidad & 11 & 27.5 \\
regular calidad & 23 & 57.5 \\
mala calidad & 6 & 15 \\
Total & 40 & 100 \\
\hline
\end{tabular}

El nivel de calidad de las anotaciones de enfermería, en la tabla 3 se aprecia que el $57.5 \%$ de anotaciones son de regular calidad.

Tabla 4

Factores personales e institucionales y el nivel de calidad de las anotaciones de enfermería del servicio de cirugía del HNGAI, Abril-Junio 2011

\begin{tabular}{|c|c|c|c|c|c|}
\hline & & & \multicolumn{3}{|c|}{ Factores asociados } \\
\hline & & & favorecen & desfavorecen & Total \\
\hline \multirow{6}{*}{$\begin{array}{l}\text { Nivel de calidad de } \\
\text { las anotaciones de } \\
\text { enfermería del ser- } \\
\text { vicio de cirugía del } \\
\text { HNGAI, Junio } 2011\end{array}$} & \multirow{2}{*}{ calidad } & $\mathrm{N}$ & 5 & 0 & 5 \\
\hline & & $\%$ & 25 & 0 & 25 \\
\hline & \multirow{2}{*}{ regular calidad } & $\mathrm{N}$ & 2 & 10 & 12 \\
\hline & & $\%$ del total & 10 & 50 & 60 \\
\hline & \multirow{2}{*}{ mala calidad } & $\mathrm{N}$ & 0 & 3 & 3 \\
\hline & & $\%$ del total & 0 & 15 & 15 \\
\hline \multirow[t]{2}{*}{ Total } & & $\mathrm{N}$ & 7 & 13 & 20 \\
\hline & & $\%$ del total & 35 & 65 & 100 \\
\hline
\end{tabular}

En cuanto a la influencia de los factores personales e institucionales en la elaboración de las anotaciones de enfermería, en la tabla 4 se puede apreciar que el 50\% de estos factores desfavorece en la elaboración de las anotaciones de enfermería, y esto se evidencia en las anotaciones de enfermería de regular calidad.

\section{DISCUSIÓN}

Los resultados presentados en la tabla 4, en cuanto a las características de las anotaciones de enfermería, reflejan la información de las anotaciones que realizaron las enfermeras del HNGAI y se observa que a pesar de que la carrera de enfermería tiene muchos años de antigüedad las enfermeras todavía tienen dificultad para realizar sus anotaciones, esto se evidenció en que el 57\% las anotaciones de enfermería fue de regular calidad.

Vargas y Ruiz (2006), en su trabajo de investigación que realizó en Costa Rica sobre aspectos legales de las notas de enfermería, muestra en sus resultados que en las notas de enfermería que se revisaron, "Se cuidó la integridad de los registros, pero se dejaron espacios en blanco en un $27 \%$, un $10 \%$ de los nombres fue ilegible. No se registró la información dada a los profesionales de salud sobre la evolución de los clientes. Esto refleja que las enfermeras todavía no superan el problema y que se siguen cometiendo los mismos errores.

La calidad de la información registrada, influye en la efectividad de la práctica profesional, por tanto los registros de enfermería deben cumplir unos estándares básicos que favorezcan la eficacia de los cuidados en las personas y legitimasen la actuación del profesional sanitario. Entonces se debe tomar muy en serio en cada institución evaluar la calidad de anotaciones de enfermería de su personal ya que como dice Hontangas (2006) "la documentación acerca de la práctica de la enfermera es necesaria para evaluar y mejorar su eficacia y productividad y es la mejor forma de evidenciar lo que hacen los enfermeros por sus pacientes en lo intelectual, emocional social y físico". 
Astonitas, Hilarion y Baca (2001), en su estudio sobre "Factores profesionales y laborales que influyen en la ejecución de las anotaciones de enfermería.

Los resultados mostraron que las anotaciones de enfermería obtuvieron un 53,3\% de ejecución regular y solo $16.3 \%$ tuvo una buena ejecución; esto se ve también en el presente trabajo de investigación y nos refiere que los factores profesionales y laborales influyen significativamente en la ejecución de adecuadas anotaciones de enfermería.

\section{CONCLUSIONES}

- Al realizar la correlación se encontró que la mayoría de factores institucionales son los que desfavorecen en la elaboración de las anotaciones de enfermería, y esto se evidencia en las anotaciones de enfermería de regular calidad.

- Al ver las características se concluye que la mayoría de las anotaciones de Enfermería en el HNGAI tienen un nivel de Calidad Regular.

\section{REFERENCIAS BIBLIOGRÁFICAS}

1. Anglade, C. (2011). Características de las anotaciones deenfermeríayfactorespersonaleseinstitucionales asociados a su elaboración en el hospital nacional “Dos de Mayo”. Recuperado de http://biblioteca. universia.net/html_bura/ficha/params/title/ caracteristicas-anotaciones-enfermería-factorespersonales-institucionales-asociados-elaboracionhospital-nacional/id/34694743.html

2. Benavent, M., Leal M. (1988). Los registros de Enfermería consideraciones ético legales. Recuperado de http://www.bioeticaweb.com/content/view/222/43/.

3. Benavent, M., Leal, M. (1988). Mesa de los aspectos Ético-Legales de los Registro de Enfermería. I Trobada d'infermeria Comunitaria. Valencia. Octubre 1998. Publicado en CB No $41,1^{\circ} 2000$.

4. Emergencias: una aplicación práctica a través de una herramienta viva. Recuperado de http://www. saludycuidados/No.7/registro/registro2.htm.
5. Martínez Ques, A.A (marzo, 2004). Aspectos legales y prácticos de los registros de enfermería. Revista Gallega de Actualidad Sanitaria. Vol. 3(1):54-59.

6. Nightingale, F. (1990). Notas sobre enfermería, qué es y qué no es. Ed. Salvat, Barcelona.

7. Romera Rufián, P. Evaluar la calidad de los registros enfermeros. Rev. Rol de Enfermería, 183: 38-47.

8. Ruiz, A. (2006). Calidad de los registros de enfermería en enfermería cardiovascular. Registro del Proceso Enfermero en el área de Urgencias y Emergencias: una aplicación práctica a través de una herramienta viva. Recuperado de http://www. saludycuidados/No.7/registro/registro2.htm.

9. Serrano, R., Sarazíbar, M., Díaz, M., Tina, P. \& Narvaiza, M. ¿Dónde comienza la calidad cientifica de los documentos de Enfermería? El reto de la documentación, 191-192: 33-40. 